\title{
Repeat OGTT at 34 - 36 Weeks to Detect the Late Occurrence of GDM: A Descriptive Cross-Sectional Study Conducted at the Professorial Unit, Teaching Hospital Peradeniya, Sri Lanka
}

\author{
Chaminda Kandauda ${ }^{1}$, W. M. M. P. B. Wanasinghe ${ }^{2}$ \\ ${ }^{1}$ Consultant Obstetrician \& Gynecologist, Department of Obstetrics \& Gynecology, University of Peradeniya, Kandy, \\ Sri Lanka \\ ${ }^{2}$ Senior Registrar, Obstetrics and Gynecology, Institute of Medicine, University of Colombo, Colombo, Sri Lanka \\ Email: chamindakandaudagynobs@gmail.com,m178.wanasinghe@gmail.com
}

How to cite this paper: Kandauda, C. and Wanasinghe, W.M.M.P.B. (2020) Repeat OGTT at 34 - 36 Weeks to Detect the Late Occurrence of GDM: A Descriptive CrossSectional Study Conducted at the Professorial Unit, Teaching Hospital Peradeniya, Sri Lanka. Advances in Reproductive Sciences, 8, 157-165.

https://doi.org/10.4236/arsci.2020.83013

Received: May 24, 2020

Accepted: June 15, 2020

Published: June 18, 2020

Copyright $\odot 2020$ by author(s) and Scientific Research Publishing Inc. This work is licensed under the Creative Commons Attribution International License (CC BY 4.0).

http://creativecommons.org/licenses/by/4.0/

\section{(c) (i) Open Access}

\begin{abstract}
Aim: Evaluation of the occurrence of glucose intolerance after 34 - 36 weeks in pregnant mothers attending the Teaching Hospital, Peradeniya. Materials \& Method: This is a descriptive cross-sectional study carried out from October 2017 to March 2018 among 183 pregnant mothers attending antenatal clinic at Teaching Hospital Peradeniya. After informed consent a repeat Oral Glucose Tolerance Test (OGTT) was done among the pregnant mothers, at period of amenorrhoea of 34 - 36 weeks, whose initial OGTT values were normal. Gestational diabetes was diagnosed according to the NICE guideline cut off values and the percentage of late occurrence of Gestational diabetes (GDM) was analyzed. Results: Mean age of the population was 29.1 years and the age range in this group was 18 to 45 years and the majority (73.9\%) of women were primipara. There were $3.3 \%$ of teenage pregnancies and $14 \%$ of mothers were over 35 years. Majority (59.7\%) of mothers underwent repeat OGTT test at 34 weeks of gestation and the rest (40.3\%) underwent test between 34 to 36 weeks of gestation. Although the mean BMI was $23.74,9.7 \%$ of mothers were obese $\left(>30 \mathrm{~kg} / \mathrm{m}^{2}\right), 30.3 \%$ of mothers were pre-obese $(25.0$ $\left.29.9 \mathrm{~kg} / \mathrm{m}^{2}\right) .8 .2 \%$ of mothers were diagnosed with Gestational diabetes at 34 36 weeks whose initial OGTT values were normal. Conclusion: In view of reducing feto-maternal morbidity and mortality, considering a repeat OGTT at a later gestation ( 34 - 36 weeks of gestation) should be considered in mothers, whose initial OGTT values are normal as Sri Lanka is a high risk
\end{abstract}


country for GDM. Clinical Significance: The study may play an important role in guideline alteration process and screening for gestational diabetes in Sri Lankan set-up.

\section{Keywords}

Gestational Diabetes, Oral Glucose Tolerance Test, Sri Lanka, Maternal and Perinatal Morbidity

\section{Introduction}

Gestational diabetes is glucose intolerance or the occurring of diabetes first recognized in pregnancy and this definition includes women with previously undiagnosed diabetes at one end of the spectrum and those with disturbances of glucose intolerance resulting from the metabolic changes in late pregnancy i.e., those above the upper limit of the normal range [1] at the other end of the spectrum. This is a common endocrine condition seen worldwide which can result in fatal neonatal outcomes and major maternal morbidities [2]. It is associated with a significantly increased risk of fetal macrosomia, shoulder dystocia, birth injuries as well as neonatal hypoglycemia and hyperbilirubinemia [3]. Even borderline GDM has been linked to an increased frequency of perinatal complications, with the maternal glycaemia demonstrating a continuous effect on perinatal outcome [4]. Babies born to mothers with GDM are at increased risk of developing obesity, diabetes and metabolic syndrome in childhood and early adulthood. Furthermore, pregnancies complicated by GDM have higher rates of caesarean section and induced deliveries with approximately 10\% - 30\% developing pre-eclampsia [3]. Women with GDM have a high risk of developing type 2 diabetes (DM) within five years postpartum with a doubling of the risk after the first five years [5].

The prevalence of GDM varies across the world with differing diagnostic criteria causing difficulty in comparing prevalence rates between geographical regions and ethnic groups. However, an increasing prevalence is noted throughout the world [6] [7] [8]. A high prevalence of GDM is reported in India, with urban (17.8\%), semi-urban (13.8\%) and rural (9.9\%) variations based on the WHO 1999 criteria [9]. The Sri Lankan data mirrors this with an approximate doubling of prevalence over seven years from $5.5 \%$ in 1998 to $10.3 \%$ in 2004 [10] [11]. Therefore, measures should be taken to increase the detection of gestational diabetes and treat in order to reduce the associated morbidity and mortality.

The Hyperglycemia and Adverse Pregnancy Outcome (HAPO) study published in 2008 was a 7-year international study that recruited 23,325 pregnant women with no prior diabetes from nine countries. Each woman underwent a 75-g oral glucose tolerance test (OGTT) between 24 and 32 weeks of gestation and was followed to the end of the pregnancy [12]. This study showed that in- 
creasing fasting glucose as well as the 1- and 2-hour post-OGTT level was all linearly correlated to adverse maternal, perinatal and neonatal outcomes.

The International Association of Diabetes in Pregnancy Study Groups (IADPSG) Consensus Panel has recommended cut-off thresholds for GDM after a 75-g OGTT between 24- and 32-weeks' gestation as follows: plasma glucose greater than $5.1 \mathrm{mmol} / \mathrm{L}$ fasting, $10.0 \mathrm{mmol} / \mathrm{L}$ at 1 hour or $8.5 \mathrm{mmol} / \mathrm{L}$ at 2 hours [13] after the HAPO study results.

The benefits of treating women who fulfill the WHO criteria for impaired glucose tolerance (IGT) between 24- and 34-week' gestation have been shown in an Australian randomized clinical trial involving 1000 women with GDM in which the perinatal outcomes were reduced [14].

Although OGTT screening was normal in mothers who were screened between 24 to 32 weeks, there were instances where macrosomic babies were delivered with the occurrence of sudden intrauterine deaths. Later investigations revealed that high blood sugar levels had been found in the mother at our clinic and the antenatal set up. Furthermore, there is literature to support that some diabetogenic hormones, especially progesterone increase near 32 weeks can elevate maternal blood glucose levels [15]. Some studies have shown that the positive predictive value of late OGTT performed at 36 - 40 weeks' gestation is 100\% [16].

Therefore, it is necessary to evaluate and study to understand whether GDM can present in later gestations although the initial OGTT is normal in order to prevent adverse outcomes of pregnancy. Since this evaluation was not done in the Sri Lankan set-up, this study aims to re-evaluate a pregnant mother with the OGTT test at $34-36$ weeks whose initial OGTT was negative when it was done around 24 - 32 weeks.

\section{Material and Methods}

The study was conducted as a cross-sectional study over 6 months from October 2017 onward at the Antenatal Clinic (ANC) and the Antenatal Ward, Teaching Hospital Peradeniya. The minimum sample size was 138 subjects with a confidence interval of 95 and a precision of 0.05 for a prevalence of $10 \%$. Hence within a period of six months, all the patients who fulfilled the inclusion criteria mentioned below and having clinic numbers of multiples of three were recruited for the study, which added up to a patient count of 182 . Information was gathered only after obtaining consent from the patient.

Pregnant women with normal initial OGTT values $(<5.6 \mathrm{mmol} / \mathrm{l}$ or 2 -hour value $<7.8 \mathrm{mmol} / \mathrm{l}$ ) were included for the study after informed consent was obtained. Pregnant women with previous diagnosis of Type 1 or 2 Diabetes mellitus, Abnormal values in 28-week OGTT and pregnant mothers who did not give consent were excluded from the study.

The selected consenting participants were screened for GDM using a 75-g 2-hour OGTT using NICE guideline criteria [17]. Subjects with fasting plasma 
glucose level of $\geq 5.6 \mathrm{mmol} / \mathrm{l}$ or 2 -hour value $\geq 7.8 \mathrm{mmol} / \mathrm{l}$ were taken as screen positive for GDM according to the above criteria [17]. The subjects' age, Period of Gestation, parity and BMI were also taken into consideration for the analysis process.

The subjects OGTT values were obtained by the team and the patient was notified regarding the results. The relevant treatments were started to prevent complications if the patient was found to be glucose intolerant.

Any patient, who wanted to withdraw voluntarily from the study due to any reason at any stage was permitted to do so. There was no loss of medical care or any other available service for leavers, which they are otherwise would have been so entitled.

Ethical clearance was obtained from the Ethical Review Committees of the Faculty of Medicine Peradeniya. Informed written consent was to be obtained. Sinhala, Tamil \& English information sheet were available to the patients. Questions and clarification were verbally explained and Investigator's details were included in the information sheet.

All the data were stored confidentially; which guaranteed that the information of the subjects was not disclosed to any third party. Only the investigators had the access to the data.

\section{Results}

A descriptive cross-sectional study was carried out from October 2017 to March 2018 among 183 pregnant mothers attending the antenatal clinic at the Teaching Hospital Peradeniya. The pregnant mothers who had normal OGTT at $24-28$ weeks were re-evaluated with OGTT at 34 - 36 weeks of period of gestation to detect the late occurrence of Gestational diabetes mellitus.

Although a majority of the pregnant mothers involved in this study were over 35 years (14\%) of age, there was a considerable amount of teenage pregnancies as well (3.3\%). The age range of the subjects spanned 18 to 45 years and the mean age was found to be 29.1 (Figure 1).

Figure 2 shows the period of gestation in which the repeat OGTT was performed to detect glucose intolerance which became apparent in late gestations in whose initial OGTT values were normal. A majority (59.7\%) had undergone testing at 34 weeks while $13.6 \%$ of mothers had undergone testing earlier than at 34 weeks of gestation. Out of the subjects, $86.4 \%$ had their reevaluation of OGTT between 34 to 36 weeks.

Figure 3 depicts the parity distribution among the mothers who had undergone OGTT reevaluation. The majority (73.9\%) were in their first and second pregnancies.

There was noticeable skewness with regard to the parity of distribution as there was progressive decline in subjects with multiparity.

There were many mothers $(30.3 \%)$ who were overweight $\left(25.0-29.9 \mathrm{~kg} / \mathrm{m}^{2}\right)$ with initial OGTT values. Moreover, $9.7 \%$ of obese (more than $30 \mathrm{~kg} / \mathrm{m}^{2}$ ) moth- 
ers also had normal initial OGTT values. Therefore, they were also included in the study expecting to have positive results during the reevaluation of OGTT to detect the late occurrence of glucose intolerance (Figure 4).

In the standard practice of Sri Lanka, according to current available guidelines, pregnant mothers are screened for glucose intolerance at their booking visit and if normal, they are reevaluated at 28 weeks of gestation prior to ruling out Gestation Diabetes Mellitus (GDM) in pregnancy. However, Figure 5 shows that there were a considerable number of mothers (8.2\%) who had become positive for glucose intolerance, while they were initially found to be screened negative for GDM according to the standard practice. This percentage of pregnant mothers may become significant when we consider feto-maternal complications arising from undetected glucose intolerance in the late gestation.

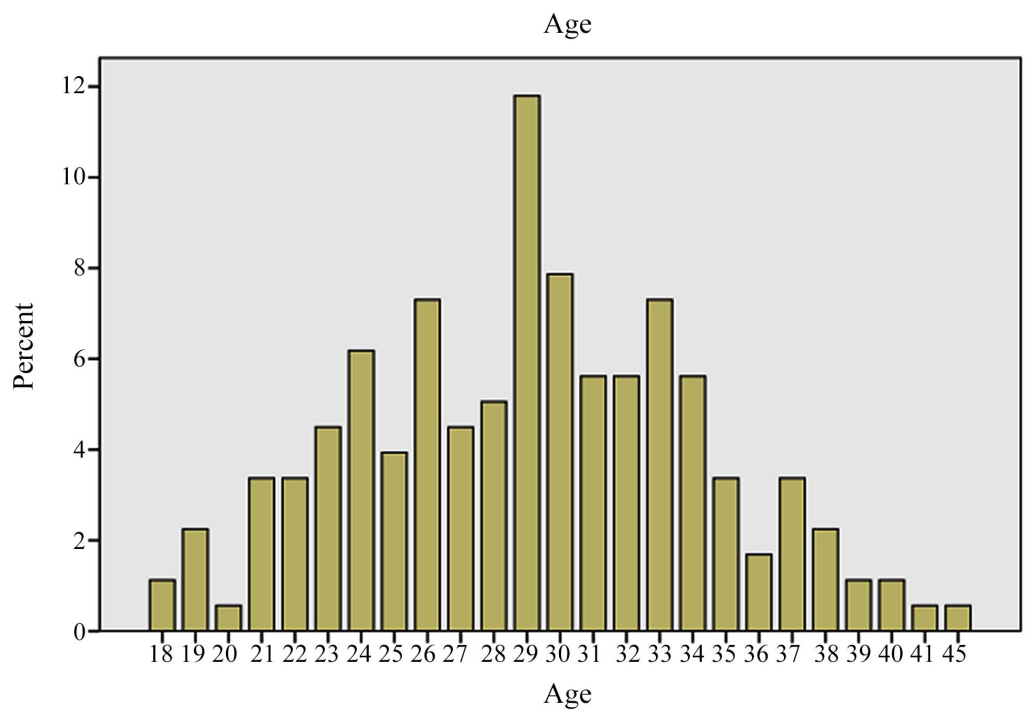

Figure 1. Age distribution of mothers of the study population.

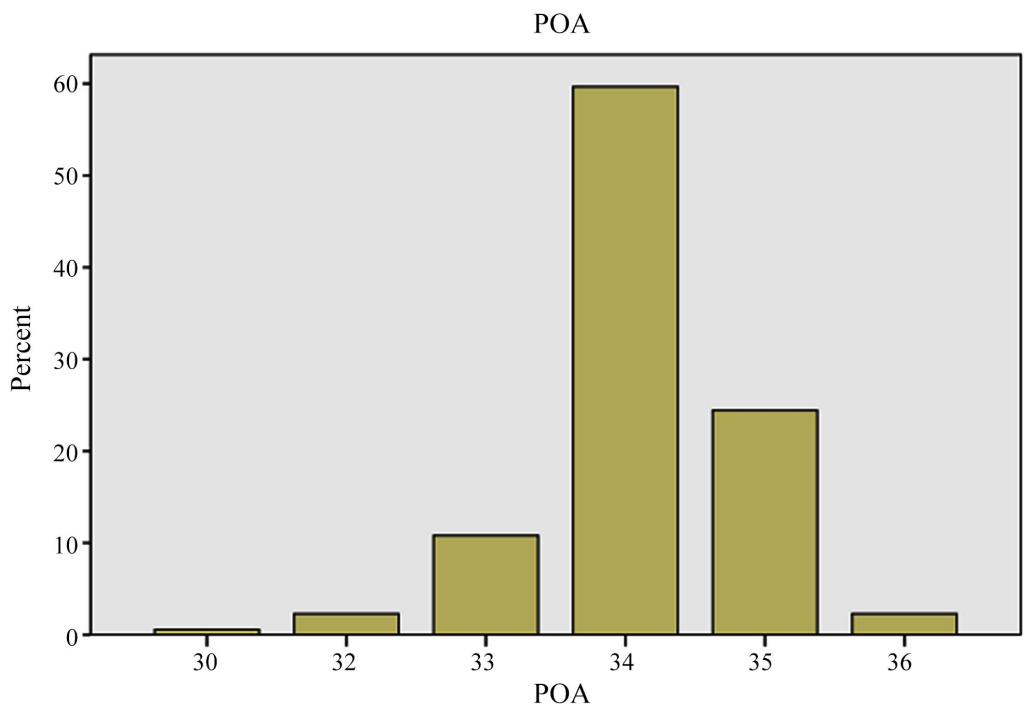

Figure 2. POA (Period of Gestation) in which re-evaluation was done. 


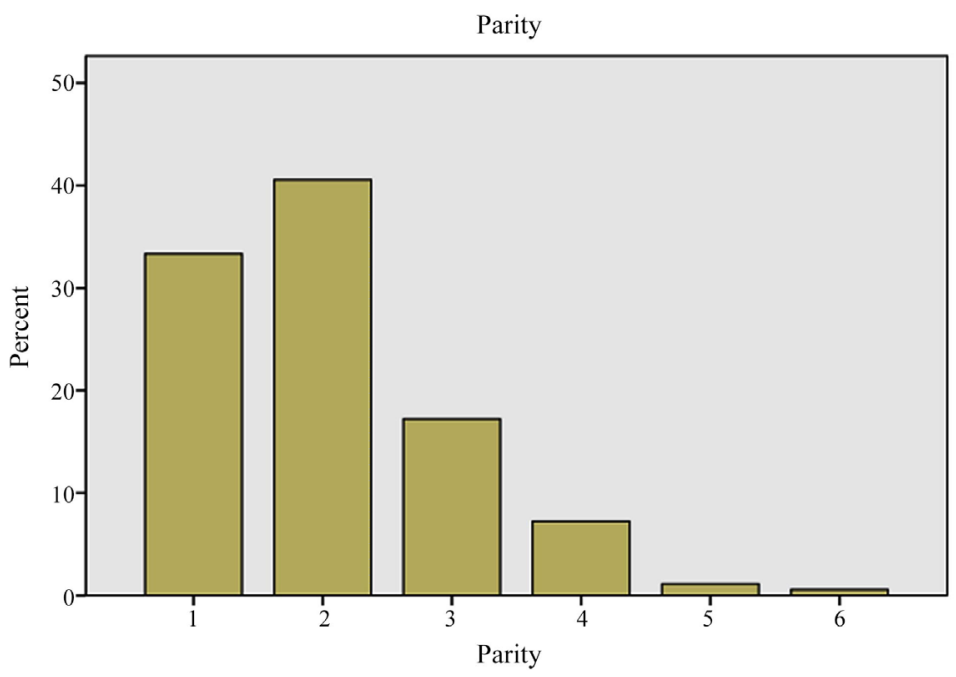

Figure 3. Parity of mothers who had undergone the OGTT re-evaluation test at $34-36$ weeks of gestation.

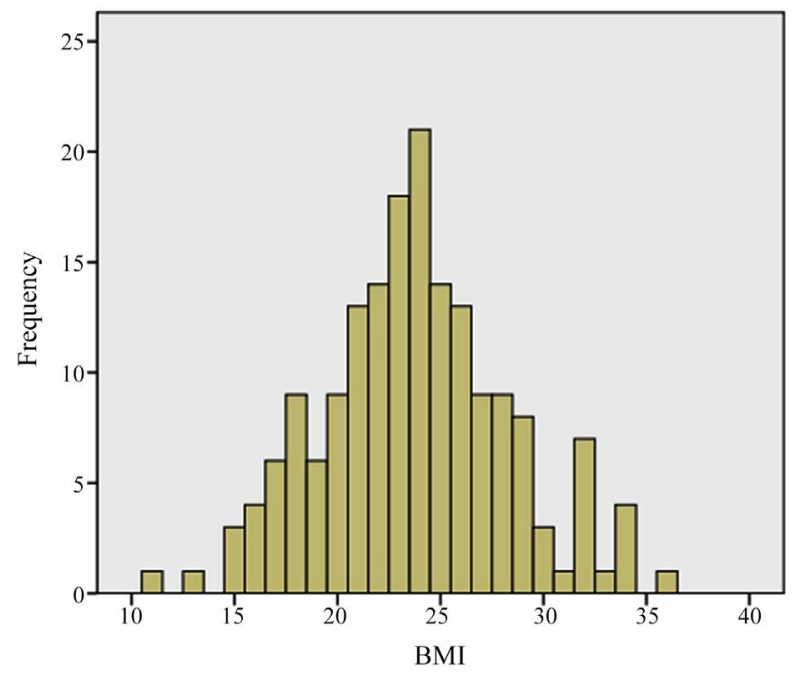

Figure 4. Frequency of BMI among the study population.

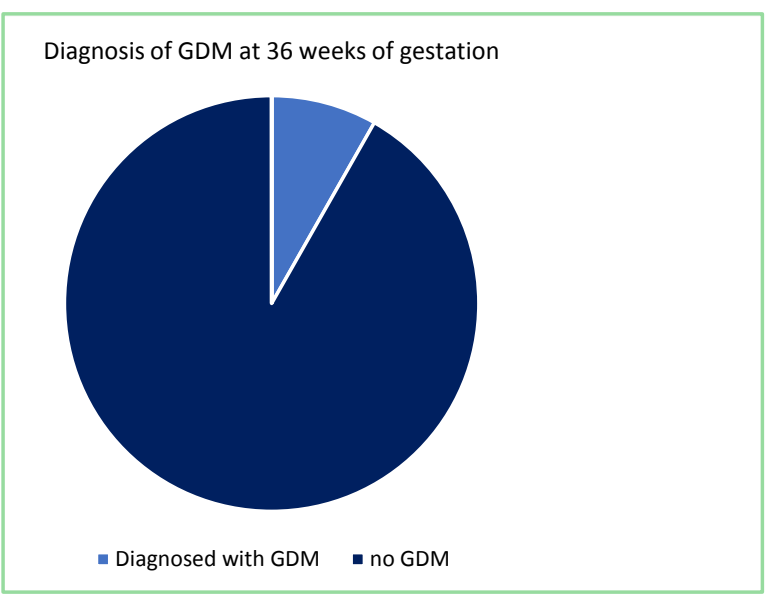

Figure 5. Percentage of mothers diagnosed with GDM using the OGTT re-evaluation test at 34 - 36 weeks of gestation. 


\section{Discussion}

It is evident from recent studies that the prevalence of GDM in Sri Lanka is increasing [18] and it is well known that adverse pregnancy outcomes are associated with gestational diabetes mellitus [19]. Therefore, if GDM is not detected by timely investigations, there will be complications during pregnancy that increase both maternal and neonatal morbidity and mortality. If stillbirths occur, it has a significant impact on the family, uncontrolled diabetes mellitus is a wellestablished risk factor for sudden still births [20].

In current practice in Sri Lanka, OGTT is done at the booking visit for those with risk factors and then it is repeated at 28 weeks for those who were negative in the initial testing. However, during investigations for unexplained stillbirths, it was found that some had undetected high blood sugar values at that time despite initially normal blood sugar values during routine screening. Therefore, the current study was conducted to assess the prevalence of GDM in the latter phase of pregnancy where the anti-insulin hormonal effect reaches a plateau whilst their initial OGTT values were normal.

In the current study, $8.2 \%$ of mothers were diagnosed with GDM, but when the OGTT was repeated at 34 - 36 weeks their initial OGTT test results were normal. In a study conducted in India, 1251 pregnant women were screened for GDM using the OGTT at the second and third trimester were found to have the prevalence rate of $18.9 \%$ [21]. Therefore, a thorough evaluation of GDM in the South East Asian population should be considered in the latter part of pregnancy, despite normal initial test results considering the higher prevalence of GDM in this segment of population.

\section{Conclusions}

Since the prevalence of GDM is high in the South East Asian countries, the risk of GDM imposed on pregnant women in Sri Lanka is considerably high [17]-[22]. Additional screening for GDM may be of value to reduce the feto-maternal complications of the late occurrence of GDM in high risk groups. These facts should be considered when designing uniform international screening and diagnostic strategies.

Our recommendation is that more studies with larger samples in different settings should be considered, to support the existing evidence prior to implementing recommendations through future guidelines.

\section{Acknowledgements}

Thank you to Mahes Salgado, the Head of the English Language Teaching Unit, University of Peradeniya for editorial assistance. I would like to extend my sincere gratitude to Professor Sampath Tennakoon, Department of Community medicine, University of Peradeniya for assisting in the analysis of data and Mr. Thejana Gunathilake, Research Assistant, Department of Gynecology and Obstetrics, University of Peradeniya for his assistance in the manuscript editing process. 


\section{Conflicts of Interest}

The authors declare no conflicts of interest regarding the publication of this paper.

\section{References}

[1] Edmonds, K. (2018) Dewhurst's Textbook of Obstetrics and Gynaecology. John Wiley \& Sons, Hoboken. https://doi.org/10.1002/9781119211457

[2] Buchanan, T.A. and Xiang, A.H. (2005) Gestational Diabetes Mellitus. The Journal of Clinical Investigation, 115, 485-491. https://doi.org/10.1172/JCI200524531

[3] Kaaja, R. and Rönnemaa, T. (2008) Gestational Diabetes: Pathogenesis and Consequences to Mother and Offspring. The Review of Diabetic Studies, 5, 194-202. https://doi.org/10.1900/RDS.2008.5.194

[4] International Association of Diabetes and Pregnancy Study Groups Consensus Panel (2010) International Association of Diabetes and Pregnancy Study Groups Recommendations on the Diagnosis and Classification of Hyperglycemia in Pregnancy. Diabetes Care, 33, 676-682. https://doi.org/10.2337/dc09-1848

[5] Bellamy, L., Casas, J.P., Hingorani, A.D. and Williams, D. (2009) Type 2 Diabetes Mellitus after Gestational Diabetes: A Systematic Review and Meta-Analysis. The Lancet, 373, 1773-1779. https://doi.org/10.1016/S0140-6736(09)60731-5

[6] Chamberlain, C., Joshy, G., Li, H., Oats, J., Eades, S. and Banks, E. (2015) The Prevalence of Gestational Diabetes Mellitus among Aboriginal and Torres Strait Islander Women in Australia: A Systematic Review and Meta Analysis. Diabetes/Metabolism Research and Reviews, 31, 234-247. https://doi.org/10.1002/dmrr.2570

[7] Leng, J., Shao, P., Zhang, C., Tian, H., Zhang, F., Zhang, S., Hu, G., et al. (2015) Prevalence of Gestational Diabetes Mellitus and Its Risk Factors in Chinese Pregnant Women: A Prospective Population-Based Study in Tianjin, China. PLoS ONE, 10, e0121029. https://doi.org/10.1371/journal.pone.0121029

[8] Chu, S.Y., Abe, K., Hall, L.R., Kim, S.Y., Njoroge, T. and Qin, C. (2009) Gestational Diabetes Mellitus: All Asians Are Not Alike. Preventive Medicine, 49, 265-268. https://doi.org/10.1016/j.ypmed.2009.07.001

[9] Seshiah, V., Balaji, V., Balaji, M.S., Paneerselvam, A., Arthi, T., Thamizharasi, M. and Datta, M. (2008) Prevalence of Gestational Diabetes Mellitus in South India (Tamil Nadu): A Community Based Study. JAPI, 56, 329-333.

[10] Siribaddana, S.H., Deshabandu, R., Rajapakse, D., Silva, K. and Fernando, D.J.S. (1998) The Prevalence of Gestational Diabetes in a Sri Lankan Antenatal Clinic.

[11] Ginege, P.S. (2004) Prevalence and Pregnancy Outcomes of Gestational Diabetes Mellitus (GDM) in Homagama DDHS Area and Validation of Selected Screening Methods to Detect GDM.

[12] Metzger, B.E., Contreras, M., Sacks, D.A., Watson, W., et al. (2008) Hyperglycemia and Adverse Pregnancy Outcomes. New England Journal of Medicine, 358, 1991-2002. https://doi.org/10.1056/NEJMoa0707943

[13] International Association of Diabetes and Pregnancy Study Groups Consensus Panel (2010) International Association of Diabetes and Pregnancy Study Groups Recommendations on the Diagnosis and Classification of Hyperglycemia in Pregnancy. Diabetes Care, 33, 676-682. https://doi.org/10.2337/dc09-1848

[14] Crowther, C.A., Hiller, J.E., Moss, J.R., et al. (2005) Effect of Treatment of Gestational Diabetes Mellitus on Pregnancy Outcomes. New England Journal of Medi- 
cine, 352, 2477-2486. https://doi.org/10.1056/NEJMoa042973

[15] Carr, D.B. and Gabbe, S. (1998) Gestational Diabetes: Detection, Management, and Implications. Clinical Diabetes, 16, 4-12.

[16] Maslovitz, S., Shimonovitz, S., Lessing, J.B. and Hochner-Celnikier, D. (2006) The Validity of Oral Glucose Tolerance Test after 36 Weeks' Gestation. European Journal of Obstetrics \& Gynecology and Reproductive Biology, 129, 19-24. https://doi.org/10.1016/j.ejogrb.2005.11.020

[17] Overview, Diabetes in Pregnancy: Management from Preconception to the Postnatal Period, Guidance, NICE. Nice.org.uk. (2015).

https://www.nice.org.uk/guidance/ng3

[18] Sudasinghe, B.H., Ginige, P.S. and Wijeyaratne, C.N. (2016) Prevalence of Gestational Diabetes Mellitus in a Suburban District in Sri Lanka: A Population Based Study. Ceylon Medical Journal, 61, 149-153. https://doi.org/10.4038/cmj.v61i4.8379

[19] Schneider, S., Hoeft, B., Freerksen, N., Fischer, B., Roehrig, S., Yamamoto, S. and Maul, H. (2011) Neonatal Complications and Risk Factors among Women with Gestational Diabetes Mellitus. Acta Obstetricia et Gynecologica Scandinavica, 90, 231-237. https://doi.org/10.1111/j.1600-0412.2010.01040.x

[20] Liu, L.C., Wang, Y.C., Yu, M.H. and Su, H.Y. (2014) Major Risk Factors for Stillbirth in Different Trimesters of Pregnancy-A Systematic Review. Taiwanese Journal of Obstetrics and Gynecology, 53, 141-145.

https://doi.org/10.1016/j.tjog.2014.04.003

[21] Seshiah, V., Balaji, V., Balaji, M.S., Sanjeevi, C.B. and Green, A. (2004) Gestational Diabetes Mellitus in India. JAPI, 52, 707-711.

[22] Nguyen, C.L., Pham, N.M., Binns, C.W., Duong, D.V. and Lee, A.H. (2018) Prevalence of Gestational Diabetes Mellitus in Eastern and Southeastern Asia: A Systematic Review and Meta-Analysis. Journal of Diabetes Research, 2018, Article ID: 6536974. https://doi.org/10.1155/2018/6536974 